\title{
ORTHOGONALITY AND NONLINEAR FUNCTIONALS ON BANACH SPACES
}

\author{
K. SUNDARESAN ${ }^{1}$
}

\begin{abstract}
If $B$ is a real Banach space and $x, y \in B$, then $x$ is said to be orthogonal to $y(x \perp y)$ if $\|x+\lambda y\| \geqq\|x\|$ for all real numbers $\lambda$. A function $F: B \rightarrow E$, where $E$ is a topological vector space, is said to be additive if it is continuous and $F(x+y)=F(x)+F(y)$ whenever $x \perp y$. The purpose of the present paper is to characterize additive functions.
\end{abstract}

If $M$ is a Banach space of real valued measurable functions on a measure space and if $x, y \in M$, then $x$ is said to be orthogonal to $y$ in the lattice theoretic sense $\left(x \perp_{L} y\right)$, if the set $\{t \mid x(t) y(t) \neq 0\}$ is of measure zero. A real valued function $F$ on $M$ is said to be $L$-additive if it is continuous, and $F(x+y)=F(x)+F(y)$ whenever $x \perp_{L} y$. Integral representations of $L$-additive functionals have been the subject of extensive study in recent years. For these and related results we refer to Drewnowski and Orlicz [1] and Sundaresan [2], and the bibliography cited therein. The concept of orthogonality in the definition of $L$-additive functionals on $M$ is very natural in these spaces. However there are several other concepts of orthogonality which have been studied in detail in arbitrary Banach spaces; see, for example, James ([3], [4]). These concepts are generalizations of orthogonality in Euclidean spaces and are of intrinsic geometric interest. One such concept of orthogonality is as follows. If $B$ is a real Banach space, and $x, y \in B$, then $x$ is said to be orthogonal to $y$, in short $x \perp y$, if $\|x+\lambda y\| \geqq\|x\|$ for all real numbers $\lambda$. The purpose of the present note is to characterize continuous real valued functions $F$ on $B$ such that $F(x+y)=$ $F(x)+F(y)$, whenever $x \perp y$. For a motivation of the study of such functionals it is enough to note that for $x, y \in L_{p}(\mu), x \perp_{L} y$ implies $x \perp y$, while in general the implication cannot be reversed.

Presented to the Society, October 31, 1970 under the title Orthogonality and nonlinear functionals on $L_{p}$-spaces; received by the editors May 19, 1970 and, in revised form, October 14, 1971.

AMS 1969 subject classifications. Primary 4610, 4615.

Key words and phrases. Banach spaces, Hilbert spaces, orthogonality, additive functions.

${ }^{1}$ The research work of the author was supported in part by a Scaife Faculty Grant (1970-71) administered by Carnegie-Mellon University.

(C) American Mathematical Society 1972 
In the rest of the paper $B$ stands for an arbitrary real Banach space of dimension at least 2 . Before proceeding to the main results of the paper we summarize useful facts concerning the concept of orthogonality $(\perp)$ described above. (1) Orthogonality is homogeneous, i.e., $x \perp y \Rightarrow \lambda x \perp \mu y$ for all real numbers $\lambda$ and $\mu$. (2) Orthogonality is not symmetric in general, i.e., $x \perp y$ does not necessarily imply $y \perp x$. However it is known, Day [5], that if the dimension of $B \geqq 3$, then orthogonality is symmetric if and only if $B$ is isometric with a Hilbert space. (3) If $x \in B$ then there exists a nonzero vector $y \in B$ such that $x \perp y$.

In passing we note that if $F$ is a continuous additive functional on $B$, then the symmetric and antisymmetric parts $F_{1}, F_{2}$ of $F$ are also continuous additive functionals on $B$. This is verified from the equations $F_{1}(x)=$ $\frac{1}{2}[F(x)+F(-x)]$, and $F_{2}(x)=\frac{1}{2}[F(x)-F(-x)]$.

We start noting three useful lemmas.

LEMMA 1. If $B$ is two-dimensional, then there are nonzero vectors $u$ and $v$ such that $u \perp v$ and $u+v \perp u-v$.

Proof. Choose arbitrary vectors $x$ and $y$ such that $x \perp y$, and $\|x\|=$ $\|y\|=1$. Represent $B$ in the plane so that $x \leftrightarrow(1,0), y \leftrightarrow(0,1)$, and $a x+$ $b y \leftrightarrow(a, b)$. Since $x \perp y$, there is a vertical line of support to the unit circle at $(1,0)$. As this line of support rotates counterclockwise, its point of support is at $(x+a y) /\|x+a y\|$, where $a$ is a continuous function of the angle of inclination. The ray from 0 to $x$-ay rotates from horizontal to the vertical, clockwise, as $a$ varies from 0 to $\infty$. Therefore there is a value of $a$ and a line of support at the corresponding point $(x+a y) /\|x+a y\|$ that is parallel to the ray from 0 to $x-a y$. That is, $x+a y \perp x-a y$. Now let $u=x$ and $v=a y$.

LEMMA 2. Let $B$ be a Banach space. If $F$ is an antisymmetric continuous additive functional on $B$, then $F$ is linear.

Proof. Let $x$ and $y$ be any two members of $B$. Choose nonzero vectors $u$ and $v$ in the linear span of $x$ and $y$ such that $u \perp v$ and $u+v \perp u-v$. By considering $F[\lambda(u+v) \pm \lambda(u-v)]$, we obtain

$$
F(2 \lambda u)=F[\lambda(u+v)]+F[\lambda(u-v)]=2 F(\lambda u),
$$

and $F(2 \lambda v)=2 F(\lambda v)$. Then by letting $\lambda=\frac{1}{2} v$, we obtain that $F\left(\frac{1}{2} v u\right)=$ $\frac{1}{2} F(v u)$. By induction, it follows that $F\left(2^{p / q} u\right)=2^{p / q} F(u)$ for all positive integers $p$ and $q$. It now follows from the continuity and antisymmetry of $F$ that $F(a u)=a F(u)$ for all real numbers $a$. Similarly $F(b v)=b F(v)$. Thus $F(a u+b v)=F(a u)+F(b v)=a F(u)+b F(v)$ so that $F$ is linear on the span of $u$ and $v$. Thus $F(a x+b y)=a F(x)+b F(y)$, for all real numbers $a$ and $b$. Hence $F$ is a linear functional on $B$. 
Lemma 3. Let $B$ be a Banach space and let $F$ be a symmetric additive functional on $B$. If $B$ is not isometric to a Hilbert space, then $F=0$. If $B$ is isometric to a Hilbert space, then there is a real number $c$ such that, for all $x \in B, F(x)=c\|x\|^{2}$.

Proof. Let $u$ and $v$ be any two members of $B$ such that $u \neq 0, u \perp v$, and $u+v \perp u-v$. Then we obtain from $F[\lambda(u+v) \pm \lambda(u-v)]$ the two equations:

$$
F(2 \lambda u)=2 F(\lambda u)+2 F(\lambda v), \quad F(2 \lambda v)=2 F(\lambda u)+2 F(\lambda v) .
$$

These equations imply that $F(2 \lambda u)=F(2 \lambda v)$ for all real numbers $\lambda$. This and the first equation imply that $F(2 \lambda u)=4 F(\lambda u)$. Then also $F\left(\frac{1}{2} v u\right)=\frac{1}{4} F(v u)$ for all $\nu$, and induction as in Lemma 2 gives

$$
F(a u)=a^{2} F(u), \text { for all } a .
$$

Similarly, $F(b v)=b^{2} F(v)$, for all $b$. Thus since $u \perp v, F(a u+b v)=a^{2} F(u)+$ $b^{2} F(v)$. Since $\lambda=\frac{1}{2}$ gives $F(u)=F(v)$, it is verified that

$$
F(a u+b v)=a^{2} F(u)+b^{2} F(v) .
$$

Suppose first that $B$ is isometric to a Hilbert space. Let $\left\{e_{\alpha}\right\}$ be an orthonormal basis of $B$. Since $e_{\alpha}+e_{\beta} \perp e_{\alpha}-e_{\beta}$ for all $\alpha$ and $\beta$, it follows from (1) that if $e=F\left(e_{\alpha}\right)$ for some specific $\alpha$ and if $x=\sum a_{i} e_{\beta(i)}$, then

$$
F(x)=F\left(\sum a_{i} e_{\beta(i)}\right)=\sum\left(a_{i}\right)^{2} F\left(e_{\alpha}\right)=\|x\|^{2} e .
$$

Next suppose that $B$ is not isometric with a Hilbert space. Then there is a two-dimensional subspace $E$ that is not isometric to Hilbert space. Now Lemma 1 assures the existence of two vectors $u, v$ such that $u \perp v, u+$ $v \perp u-v$, and the linear span of $u, v$ is $E$. Let now $E$ be represented in the plane by $u \leftrightarrow(1,0)$ and $v \leftrightarrow(0,1)$. Then there is a line of support $L$ to the unit circle at a point $(\alpha u+\beta v) /\|\alpha u+\beta v\|$, for which $L$ is not perpendicular to the ray from the origin to $\alpha u+\beta v$. Thus $a u+\beta v \perp \beta u-c v$ where $c \neq \alpha$, and the rays from the origin to $(\alpha, \beta)$ and $(\beta,-c)$ are not perpendicular. Then

$$
\begin{aligned}
F\left[\left(c \alpha+\beta^{2}\right) u\right] & =F[c(\alpha u+\beta v)+\beta(\beta u-c v)] \\
& =F(c \alpha u+c \beta v)+F\left(\beta^{2} u-\beta c v\right) .
\end{aligned}
$$

It then follows from (1) that $\left(c \alpha+\beta^{2}\right)^{2}=c^{2} \alpha^{2}+c^{2} \beta^{2}+\beta^{4}+\beta^{2} c^{2}$ and $c \alpha \beta^{2}=c^{2} \beta^{2}$. Similarly, $c \alpha \beta^{2}=\alpha^{2} \beta^{2}$. Since $c \neq \alpha$, it follows that $\beta=0$. This is impossible, since $(\alpha, 0)$ is not orthogonal to $(0,-c)$ unless $c=0$. This completes the proof of the lemma.

We now proceed to the main result of the paper characterizing continuous additive functionals on a Banach space. We state the result in the more general setting, allowing the additive function to take values in a locally convex space. 
THEOREM 1. Let $F$ be a continuous function on a Banach space $B$ into a locally convex space $E$. If $B$ is a Hilbert space, then $F$ is additive if and only if there exist a vector $\xi \in E$ and a continuous linear operator $T: B \rightarrow E$ such that $F(x)=\|x\|^{2} \xi+T(x)$ for all $x \in E$. Further if $B$ is not isometric with $a$ Hilbert space, then $F$ is a continuous linear operator on $B$ to $E$.

Proof. Let $B$ be a Hilbert space. Let $E^{*}$ be the topological dual of $E$. Let $F$ be an additive function on $B \rightarrow E$. Let $F_{1}, F_{2}$ be the symmetric and antisymmetric parts of $F$. Now if $f \in E^{*}$, then $f \circ F_{1}$ and $f \circ F_{2}$ are symmetric and antisymmetric additive functionals on $B$. Thus from Lemmas 2 and 3 , it is inferred that there are a constant $c_{f}$ and a continuous linear functional $l_{f}$ on $B$ such that $f \circ F_{1}(x)=c_{f}\|x\|^{2}$ and $f_{\circ} F_{2}(x)=l_{f}(x)$. It is verified that the mapping $f \rightarrow c_{f}$ is an $\omega^{*}$-continuous linear functional on $E^{*}$. Hence there exists a fixed vector $\xi \in E$ such that $f(\xi)=c_{f}$ for all $f \in E^{*}$. Since $E^{*}$ separates points in $E$, it follows that $F_{1}(x)=\|x\|^{2} \xi$. Again, since for each $f \in E^{*}, f \circ F_{2}$ is a linear functional on $B$, it follows that $F_{2}$ is a linear operator on $B \rightarrow E$. Further, since $F$ is continuous, $F_{2}$ is a continuous linear operator on $B \rightarrow E$. Considering symmetric and antisymmetric parts of $F$ it is verified that $\xi$ and $T$ are uniquely determined by $F$. Thus $F(x)=\|x\|^{2} \xi+T(x)$ as stated in the theorem. Since any function $F$ with the above representation is an additive function, the proof of the part of the theorem dealing with the case when $B$ is a Hilbert space is complete. The case when $B$ is not a Hilbert space is similarly dealt with, and the proof is omitted.

ACKNOWLEDGEMENT. The present note is a revised version of the paper Orthogonality and nonlinear functionals on $L_{p}$-spaces. Theorem 1 in this note was proved in the earlier paper for the case when $B$ is an $L_{p}$-space, $1 \leqq p \leqq \infty$. The present version of the theorem for all Banach spaces $B$, $\operatorname{dim} B \geqq 2$, is essentially due to the referee. I am extremely grateful to the referee for this and several other comments.

\section{REFERENCES}

1. L. Drewnowski and W. Orlicz, On orthogonally additive functionals, Bull. Acad. Polon. Sci. Sér. Sci. Math. Astronom. Phys. 16 (1968), 883-888. MR 39 \#6069.

2. K. Sundaresan, Additive functionals on Orlicz spaces, Studia Math. 32 (1969), 269276. MR $40 \# 1765$.

3. R. C. James, Orthogonality in normed linear spaces, Duke Math. J. 12 (1945), 291302. MR 6, 273.

4. - Orthogonality and linear functionals in normed linear spaces, Trans. Amer. Math. Soc. 61 (1947), 265-292. MR 9, 42.

5. M. M. Day, Some characterizations of inner-product spaces, Trans. Amer. Math. Soc. 62 (1947), 320-337. MR 9, 192.

Department of Mathematics, Carnegie-Mellon University, Pittsburgh, PenNSYlVania 15213 